\title{
Successful Application of Transoral Robotic Surgery in Failures of Traditional Transoral Laser Microsurgery: Critical Considerations
}

\author{
Claudio Vicini $^{a} \quad$ Carlo Antonio Leone $^{b}$ Filippo Montevecchi ${ }^{a}$ \\ Elisa Dinellic Veronica Secciad Iacopo Dallan ${ }^{d}$ \\ ${ }^{a}$ Oral Surgery Unit, Otolaryngology - Head and Neck Surgery Division, Department \\ of Special Surgery, G.B. Morgagni L. Pierantoni Hospital, Forli, b ${ }^{\text {Department of }}$ \\ Otorhinolaryngology - Head and Neck Surgery Division, Monaldi Hospital, Naples, \\ 'Otorhinolaryngology Unit, 'S. Camillo' Clinic, Forte dei Marmi, and d Department of \\ Otorhinolaryngology, University of Pisa, Pisa, Italy
}

\section{Key Words}

Transoral robotic surgery · Difficult laryngeal exposure $\cdot$ Transoral laser microsurgery failures

\begin{abstract}
Aims: To assess the role of transoral robotic surgery (TORS) in managing failures of conventional transoral laser microsurgery (TLM) in cases with difficult laryngeal exposure. Methods: Retrospective analysis of 3 patients with difficult laryngeal exposure treated with TORS. All 3 cases failed to be managed by conventional TLM after repeated attempts by experienced surgeons. In 2 cases, the initial disease was laryngeal cancer treated by a cricohyoidoepiglottopexy, with an obstructing residual epiglottis. The last case was a bilateral Reinke disease submitted to repeated TLM procedures, producing multiple supraglottic and glottic scars. In all cases, the difficult exposure was produced by a combination of concurrent elements including insufficient mouth opening, short and stiff neck, macroglossia and high-positioned larynx. Results: Two patients were exposed by means of a Davis Meyer mouth gag. The other patient was managed by a Feyh-Kastenbauer device. The key of the success was the possibility to work 'around the corner' ( $30^{\circ}$ angle view) where straight alignment of the larynx was impossible. Conclusions: Where possible, a TORS approach should be considered complementary to TLM in cases of very difficult or even impossible conventional transoral laryngoscopic approach.
\end{abstract}




\section{Introduction}

Since their dawn at Penn's University [1,2], robot-assisted procedures have been applied in many head and neck areas, and actual medical literature witnesses this amazing evolution. The daVinci ${ }^{\circledR}$ surgical system (Intuitive Surgical, Inc., San Francisco, Calif., USA) is currently used in oral cavity, orohypopharyngeal, laryngeal, parapharyngeal and thyroidal regions, both for benign and malignant diseases [3-5]. Indications for the robotic approach in the head and neck area are increasing day by day. More recently, also the neck and submandibular regions have been addressed in preliminary series by means of a face-lift approach [6], and even reconstructive surgery is now increasingly using the daVinci technology [7-10]. Currently, infratemporal and skull base regions have been explored in preclinical studies [11-15].

Similarly, in any other minimally invasive approach, the most critical aspect in transoral robotic surgery (TORS) is the necessity to adequately expose the surgical field. Furthermore, the surgical space must be large enough to handle the anatomic structures by means of the robotic arms without conflict between them. In simple words, the feasibility of any given procedure is one of the first elements to be evaluated [16,17]. In this respect, with the aim of reducing the risk of prematurely ending the robotic procedure, several tricks have been described and proposed in order to increase the ability to adequately expose and manage the area of interest [18]. This merely practical aspect of the robotic procedures is stressed in all papers dealing with TORS, where the conversion rate towards traditional microscopic laserassisted or open procedures is always reported. On the other hand, even traditional laryngoscopic exposure may not be feasible due to technical and/or anatomical problems. Risk factors for difficult laryngeal exposure have been described [19]. Among these, obesity has been found as one of the most critical elements in predicting difficult exposure [20]. As previously stated, to perform TORS safely, an adequate oral access is mandatory. Consequently, this kind of procedure can be proposed in selected cases, and one of the most critical elements to be evaluated is the interincisive distance. Currently, robotic techniques are considered alternative to traditional microscopic procedures in selected cases. Herein, we report our clinical experience in which a robot-assisted procedure has allowed us to manage conditions not suitable for traditional procedures, thus overcoming the limits of microscope-assisted procedures. Our study cohort comprised 3 cases that represent the subjects of debate. This retrospective analysis was approved by the local Ethical Committee of the G.B. Morgagni L. Pierantoni Hospital. All patients were informed and gave their written consent. A short summary of the patients' data is given in table 1 .

\section{Case Report}

Case 1

Case 1 was a 62-year-old male who underwent supracricoid partial laryngectomy with cricohyoidoepiglottopexy with left arytenoidectomy and selective II-IV-level omolateral neck dissection for a moderately differentiated squamous cell carcinoma (SCC) of the left vocal cord (cT2 N0 M0) on September 13, 2010. The pathological stage was pT1a N0 M0, and the surgical margins were negative. The patient was decannulated 4 months later for the presence of postsurgical edema. Furthermore, 2 months later, dyspnea was still present due to persistent edema of the right arytenoid and residual epiglottic cartilage. Tracheotomy was performed and laser $\mathrm{CO}_{2}$ resection and vaporization of the exuberant tissues was attempted. However, the procedure failed, given the impossibility to reach and adequately expose the anterior aspect of the neolarynx by transoral laser microsurgery (TLM). Failure was principally due to a combination of a high-positioned larynx, stiff neck, micrognathia and Cormack and Lehane grading 4. A complete opening of the airways was not possible. 
Vicini et al.: Successful Application of Transoral Robotic Surgery in Failures of

Traditional Transoral Laser Microsurgery: Critical Considerations

Table 1. Detailed data of the patient cohort

\begin{tabular}{|c|c|c|c|c|c|c|c|c|}
\hline Patient & Age, years & Sex & $\begin{array}{l}\text { First surgical } \\
\text { procedure }\end{array}$ & $\begin{array}{l}\text { Compli- } \\
\text { cations }\end{array}$ & TLM limitations & TORS procedure & $\begin{array}{l}\text { Swallowing } \\
\text { complications } \\
\text { after TORS }\end{array}$ & $\begin{array}{l}\text { Respiratory } \\
\text { problems after } \\
\text { TORS }\end{array}$ \\
\hline 1 & 62 & M & $\begin{array}{l}\text { CHEP with left } \\
\text { arytenoidectomy } \\
\text { and omolateral } \\
\text { neck dissection } \\
\text { (levels II-IV) }\end{array}$ & Dyspnea & $\begin{array}{l}\text { High-positioned } \\
\text { larynx, stiff neck, } \\
\text { micrognathia } \\
\text { and Cormack } \\
\text { and Lehane } \\
\text { grading } 4\end{array}$ & $\begin{array}{l}\text { Residual obstructing } \\
\text { epiglottic fold } \\
\text { resection }\end{array}$ & - & - \\
\hline 2 & 56 & M & $\begin{array}{l}\text { CHEP and bilateral } \\
\text { neck dissection } \\
\text { (levels II-IV) }\end{array}$ & Dyspnea & $\begin{array}{l}\text { High-positioned } \\
\text { larynx and stiff } \\
\text { neck }\end{array}$ & $\begin{array}{l}\text { Complete } \\
\text { epiglottectomy }\end{array}$ & - & - \\
\hline 3 & 73 & $\mathrm{~F}$ & $\begin{array}{l}\text { Laser } \mathrm{CO}_{2} \\
\text { cordotomy after } \\
\text { thyroidectomy }\end{array}$ & Dyspnea & $\begin{array}{l}\text { Cormack and } \\
\text { Lehane grading } \\
3 \text {, micrognathia }\end{array}$ & $\begin{array}{l}\text { TBR and } \\
\text { epiglottoplasty, } \\
\text { partial bilateral } \\
\text { arytenoidectomy, } \\
\text { posterior right } \\
\text { cordotomy, releasing } \\
\text { of strictures }\end{array}$ & - & - \\
\hline
\end{tabular}

CHEP = Cricohyoidoepiglottopexy; TBR = tongue base reduction.

In this first case, a TORS approach was attempted and performed on April 30, 2012. With a Davis Meyer mouth gag (short blade) and the anterior retraction of the tongue, an adequate exposure of the neolarynx was obtained. Thus, the residual obstructing epiglottic folds were managed by means of angled robotic arms under a $30^{\circ}$ endoscopic view. The feeding tube was not inserted. The tracheal cannula was removed 1 month postoperatively. Currently, at 10 months postoperatively, the patient does not present any respiratory problem or swallowing complications.

Case 2

Case 2, a 56-year-old male, presented to our department with a right cordo-commissural, well-differentiated squamous cell carcinoma and a clinical suspicion of bilateral cervical node invasion (T1a/N2c/M0). On January 31, 2012, he underwent a supracricoid partial laryngectomy with cricohyoidoepiglottopexy and bilateral selective neck dissection (levels II-IV). Definitive staging was pT1a/N0/M0 with negative margins. At 3 months postoperatively, the patient was still unable to breathe through the normal airways. An attempt to manage bulky and scar tissues was done by means of TLM but was unsuccessful, given the impossibility to visualize the area to be treated. Similar to the previous case, failure was principally due to a combination of a high-positioned larynx, stiff neck and micrognathia.

The patient was treated with an epiglottectomy by means of a TORS approach on April 3, 2012. The Feyh-Kastenbauer retractor was used to expose the supraglottic area for TORS, and a resection of the whole epiglottis was done (fig. 1). At 10 months postoperatively, the patient did not present any respiratory problem or swallowing complications.

Case 3

A 73-year-old female had a bilateral vocal fold paresis after thyroidectomy. In November 2012, a $\mathrm{CO}_{2}$ laser endolaryngeal cordotomy was performed elsewhere. Given the persistence of respiratory distress, a tracheotomy was performed on May 5, 2012. An endoscopic preoperative evaluation showed an interarytenoid and supraglottic stenosis with significantly reduced respiratory space. A significant tongue base hypertrophy (Cormack and Lehane grading 3) associated with micrognathia prevented proper laryngeal exposure by means of the traditional microscopic approach. In this last case, TORS was performed on June 11, 2012, using a Davis Meyer mouth gag (medium blade). A tongue base reduction and an epiglottoplasty were needed to better visualize the surgical field. Partial bilateral arytenoidectomy, posterior right cordotomy 

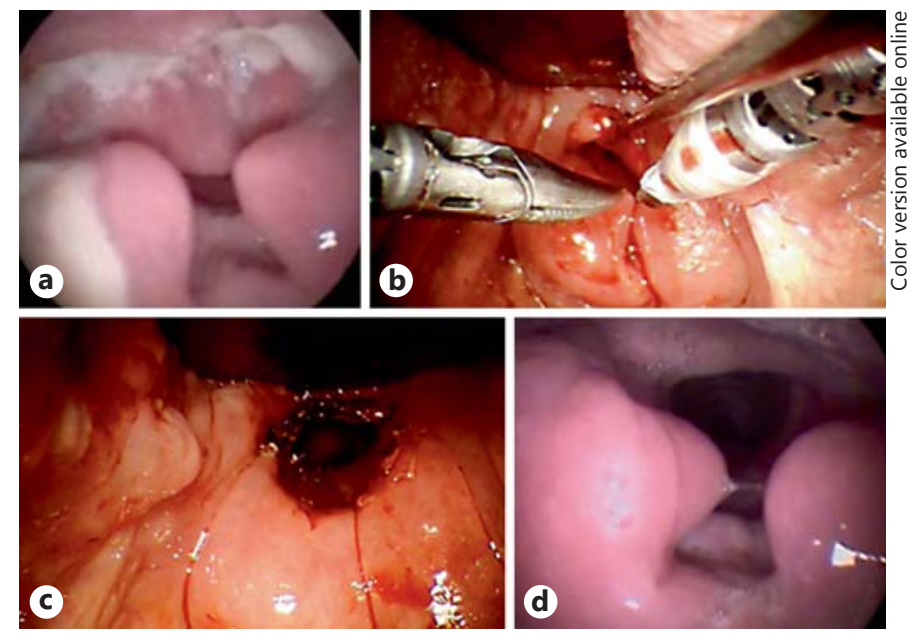

Fig. 1. a Preoperative laryngoscopy. In the superior area of the neolarynx, a severely retrodisplaced epiglottis tilts back, reducing the airway below the level of the arytenoids. $\mathbf{b}$ TORS epiglottectomy. From top to bottom: the blade is supporting the tongue base, and the epiglottis is grasped by a forceps. On the left: a 5-mm Maryland robotic forceps; on the right: a 5-mm blunt tip monopolar instrument; at the bottom: both arytenoids are visible. $\mathbf{c}$ The surgical bed at the end of the TORS procedure after the complete removal of the epiglottis. d The larynx 3 months after surgery.
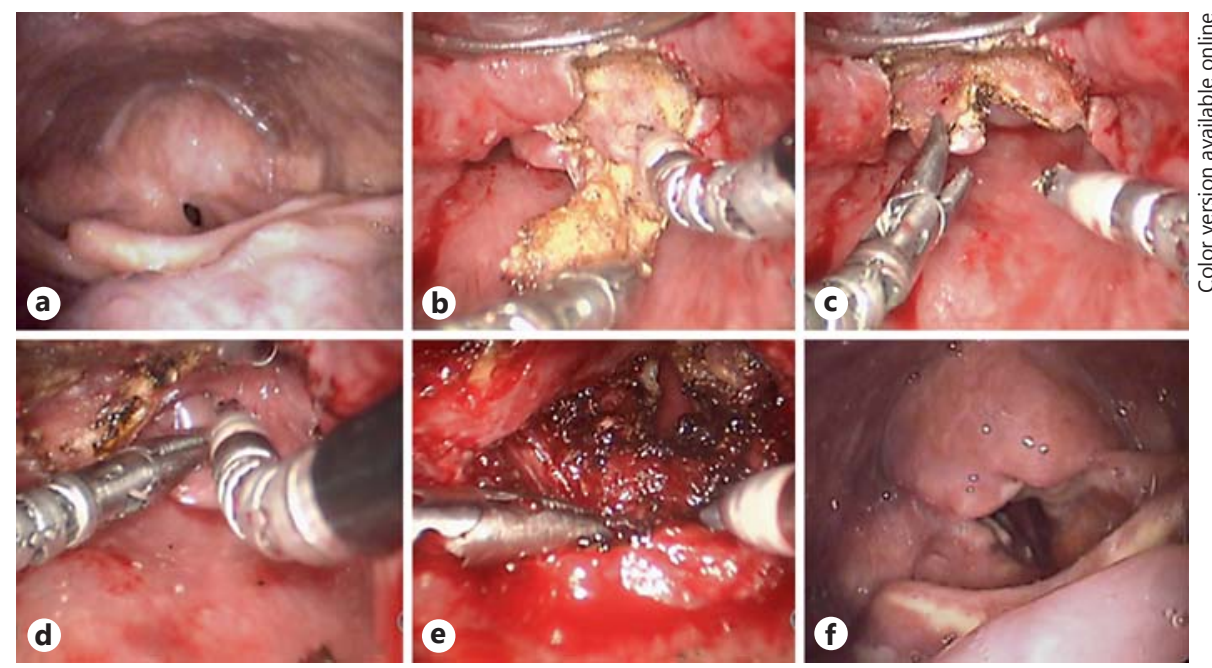

Fig. 2. a Preoperative laryngoscopy. Interarytenoid and supraglottic strictures and hypertrophic tongue base (Cormack and Lehane grading 3). b Tongue base reduction. From top to bottom: the blade is supporting the tongue base, and the tongue base is grasped by a forceps. On the left: a 5-mm Maryland robotic forceps; on the right: a 5-mm blunt tip monopolar instrument. c TORS epiglottoplasty. d, e Partial bilateral arytenoidectomy and releasing of supraglottic strictures. $\mathbf{f}$ The larynx 3 months after surgery.

and scar removal were then successfully performed (fig. 2). At the end of the procedure, a gauze spacer was placed between the residual arytenoids and was removed 2 days later. The procedure was successfully performed, and 7 months later, the tracheostomy tube was removed. Currently, the patient does not present any respiratory distress or swallowing complications. As a whole, the TORS approach was performed successfully in all cases. The mean follow-up was 9 months, with a range from 7 to 10 months. No serious complications have been observed in our series, and no revision surgery was deemed necessary in any case. 
Vicini et al.: Successful Application of Transoral Robotic Surgery in Failures of

Traditional Transoral Laser Microsurgery: Critical Considerations

\section{Discussion}

In the last few years, TORS has been proved a sound endoscopic approach in selected upper airways diseases. Since its birth in the mid years of the last decade, amazing experience has been gained, and the US Food and Drug Administration (FDA) granted the use of the daVinci surgical system for transoral surgery in otolaryngology, namely for benign and malignant lesions (T1T2) of the oropharynx, in December 2009. Beyond the continuously growing number of medical publications, the widespread application of TORS worldwide witnesses the feasibility, safety and efficacy of robot-assisted procedures. Notwithstanding, since its introduction, one of the main concerns expressed about TORS has been its feasibility. The presence of bulky instruments and the conflict with the access window have been indicated as some of the main problems with this kind of surgery. Consequently, even now, in the majority of the papers focused on TORS, an evaluation of the conversion rate (towards TLM or open procedures) is presented and assessed, thus showing the necessity to demonstrate the applicability of the procedure.

Given the fact that surgery is a matter of vision, a surgically adequate exposure of the field is one of the most important mainstays. In TORS, the exposure of the area of interest may be a challenge. An accurate preoperative evaluation under general anesthesia with a $30^{\circ}$ endoscope and a Feyh-Kastenbauer retractor can help minimize the number of robotic cases interrupted intraoperatively. Obviously, as in any other minimally invasive approach, the correct exposure of the lesion is a mandatory condition for the success of the procedure, and in TORS, a good exposure means not only a complete view of the lesion but also sufficient space for the movements of the robotic arms. In this respect, many tricks and solutions have been described in order to increase the ability of adequately exposing and managing the area of interest [18]. For example, manual posterior compression of the larynx can improve the exposure of the anterior commissure, and even the anterior placement of the tongue is a simple and effective maneuver that can be very useful in cases where the footprint of the tongue does not allow a correct view of the instruments. Regarding the topic of arm collisions, we can state that according to our experience, such problems can be significant when dealing with hypopharyngeal regions but are much less severe in supraglottic or glottis procedures. Anyway, we maintain that a careful robotic setting using adequate tricks (previously described), meticulous dissection and appropriate assistance by the second surgeon helps avoiding conflicts between the arms. Furthermore, the use of noncontact lasers allows cutting without the need to touch the tissues, thus reducing the necessity to have both the instruments' tips handling the target. The use of such technical refinements can increase the feasibility of the procedure, especially in very complex situations.

From a clinical point of view, many areas of the head and neck have been addressed by means of a TORS approach, with the oropharynx, laryngohypopharynx, parapharynx and thyroid being among the most commonly and frequently treated sites [1-5]. More recently, robotassisted procedures have also been applied to submandibular and neck regions [6]. Similar to any other procedure, TORS can be performed in previously selected cases. As far as TORS is concerned, the most limiting aspect is the adequate access to the area of interest. We agree with Weinstein and O'Malley [21] that all primary tongue base, larynx and hypopharyngeal lesions should undergo a separate staging endoscopy in order to assess whether adequate exposure for TORS can be achieved. On the other hand, it is also well known that TLM cannot be performed in every case. Although TLM is universally considered the gold standard in endoscopic laryngeal surgery, all endoscopic laryngeal surgeons remember cases in which an adequate laryngeal exposure is not an achievable goal. When the endolarynx is not fully visualized in a given patient, a combination of concomitant situations usually occurs. A reduced interincisive distance may be the first obstacle, and a wide tongue body is another common finding. However, a short and stiff neck can be a limiting factor as well. In patients treated by means of a partial laryngectomy, a combination of a high-positioned larynx and a stiff neck may be of great concern. 
From a surgical point of view, the basic prerequisite for a good TLM is the ability to control the upper airways; in other and simpler words, it is mandatory to obtain a completely 'straight' cavity. In such cases, a direct microscopic visualization is possible, and at the same time, a rectilinear laser beam can be delivered and 'straight' microinstruments can be used. The main limit of TLM is the impossibility to deal with laryngeal areas that are out of direct sight, hidden or placed 'around the corner'. In such situations, TORS may be a rescue option and, given its intrinsic features, it should be considered complementary and not alternative to TLM. In fact, it is well known that the robotic technology provides unique advantages by allowing an angled 3D visualization of the surgical field, instrumentations with multiple degrees of rotation and increased surgical accuracy and precision. TORS may overcome some restrictions of TLM related to the obliged 'line-of-sight' working space. In our case, a $30^{\circ}$ faceup 8-mm scope has proved to be an adequate tool for wide laryngeal visualization. A complete straightening of all upper airways is not required. In our setting, an open blade is preferred to a closed tube for larynx exposure. It allows the introduction of a couple of 5-mm snake instruments with so-called 'EndoWrist ${ }^{\circledR}$ ' movement capabilities. From a practical point of view, this means the ability to work around the corner, under the control of a $30^{\circ}$ tridimensional scope. These wristed ends also allow for surgeries that are highly precise and provide the advantage of multiplanar resection. Consequently, the combination of an 'around-thecorner' view and precise manipulation has proved to be the key of the success of TORS in our TLM failures. Not of minor importance, the daVinci system also allows the surgeon to assume a comfortable position during the procedure and prevents tremor using both the instruments and the camera [22].

Among the known drawbacks of the daVinci technology that are still in the process of resolution, we mention the lack of 'natural' tactile feeling [23] and the availability of suction instruments. Notwithstanding, in our experience, they do not prevent the successful outcome of the procedures. Our experience seems to demonstrate that TORS, with its own particular features, is possible even in cases where TLM has failed to obtain an adequate exposure. In our patients, the straight alignment of the larynx was impossible, and very skilled and experienced surgeons were not able to obtain an adequate exposure. The use of a nontubular blade made it possible to introduce a couple of 5-mm snake arms capable of manipulating the larynx around the corner. Although preliminary, our experience seems to demonstrate that, if available, a TORS approach should be considered as a rescue option in cases of very difficult or even impossible conventional TLM. Although we are perfectly aware of the subjectivity of our statements, we are at the same time convinced that our experience is worth being brought to attention, as we do not consider our cases to be that rare. Larger studies and multicenter experience will confirm our personal point of view.

\section{Conclusions}

In selected cases, TORS proved to be a possible salvage option for TLM failures. In difficult cases of laryngeal exposure, the classic closed laryngoscope may fail to create a rectilinear cavity between the microscope and the larynx. In such cases, the wide exposure provided by the use of a mouth gag coupled with a movable wide-angle system, the motion capacity of the robotic instruments and high-definition 3D cameras of the daVinci platform may overcome the problem in a very simple and quick way. In simpler words, in our experience, the success of TORS was determined by the possibility of working 'around the corner', using curved lenses and angled instruments. Based on this and on the growing experience, we strongly maintain that TORS is worth being included in the armamentarium of any head and neck surgeon. Additionally, it could be applied even in cases not amenable to traditional micro- 
Vicini et al.: Successful Application of Transoral Robotic Surgery in Failures of

Traditional Transoral Laser Microsurgery: Critical Considerations

scopic approaches. The development of smaller robotic arms and instrumentations as well as further advances and modifications such as reliable integrated flexible laser technology will increase the applicability of such technology in the otolaryngology field.

\section{Acknowledgment}

We thank Mrs. Diana Elizabeth Hearn for her help in translating and revising the paper.

\section{Disclosure Statement}

The authors have no conflicts of interest to disclose.

\section{References}

1 Hockstein NG, Nolan JP, O’Malley BW Jr, et al: Robotic microlaryngeal surgery: a technical feasibility study using the daVinci surgical robot and an airway mannequin. Laryngoscope 2005;115:780-785.

2 Hockstein NG, Nolan JP, O'Malley BW Jr, et al: Robot-assisted pharyngeal and laryngeal microsurgery: results of robotic cadaver dissections. Laryngoscope 2005;115:1003-1008.

- 3 O'Malley BW Jr, Quon H, Leonhardt FD, et al: Transoral robotic surgery for parapharyngeal space tumors. ORL J Otorhinolaryngol Relat Spec 2010;72:332-336.

- 4 Arshad H, Durmus K, Ozer E: Transoral robotic resection of selected parapharyngeal space tumors. Eur Arch Otorhinolaryngol 2013;270:1737-1740.

- 5 Dallan I, Montevecchi F, Seccia V, Vicini C, Segnini G, Casani AP, Sellari-Franceschini S: Transoral robotic resection of an ectopic tongue-base thyroid gland. J Robot Surg 2013;7:83-86.

6 De Virgilio A, Park YM, Kim WS, et al: Robotic sialoadenectomy of the submandibular gland via a modified facelift approach. Int J Oral Maxillofac Surg 2012;41:1325-1329.

7 Mukhija VK, Sung CK, Desai SC, et al: Transoral robotic assisted free flap reconstruction. Otolaryngol Head Neck Surg 2009;140:124-125.

- 8 Genden EM, Kotz T, Tong CC, et al: Transoral robotic resection and reconstruction for head and neck cancer. Laryngoscope 2011;121:1668-1674.

- 9 de Almeida JR, Genden EM: Robotic assisted reconstruction of the oropharynx. Curr Opin Otolaryngol Head Neck Surg 2012;20:237-245.

$10 \mathrm{Kim}$ CH, Chang JW, Choi EC, et al: Robotically assisted selective neck dissection in parotid gland cancer: preliminary report. Laryngoscope 2013;123:646-650.

11 Lee JY, O'Malley BW Jr, Newman JG, et al: Transoral robotic surgery of the skull base: a cadaver and feasibility study. ORL J Otorhinolaryngol Relat Spec 2010;72:181-187.

12 McCool RR, Warren FM, Wiggins RH 3rd, et al: Robotic surgery of the infratemporal fossa utilizing novel suprahyoid port. Laryngoscope 2010;120:1738-1743.

13 Dallan I, Castelnuovo P, Seccia V, et al: Combined transnasal transcervical robotic dissection of posterior skull base: feasibility in a cadaveric model. Rhinology 2012;50:165-170.

14 Kim GG, Zanation AM: Transoral robotic surgery to resect skull base tumors via transpalatal and lateral pharyngeal approaches. Laryngoscope 2012;122:1575-1578.

15 O’Malley BW Jr, Weinstein GS: Robotic skull base surgery: preclinical investigations to human clinical application. Arch Otolaryngol Head Neck Surg 2007;133:1215-1219.

16 Hockstein NG, O’Malley BW Jr, Weinstein GS: Assessment of intraoperative safety in transoral robotic surgery. Laryngoscope 2006;116:165-168.

17 Weinstein GS, O’Malley BW Jr, Magnuson JS, et al: Transoral robotic surgery: a multicenter study to assess feasibility, safety, and surgical margins. Laryngoscope 2012;122:1701-1707.

18 De Virgilio A, Park YM, Kim WS, et al: How to optimize laryngeal and hypopharyngeal exposure in transoral robotic surgery. Auris Nasus Larynx 2012;40:312-319.

19 Pinar E, Calli C, Oncel S, et al: Preoperative clinical prediction of difficult laryngeal exposure in suspension laryngoscopy. Eur Arch Otorhinolaryngol 2009;266:699-703.

20 Hekiert AM, Mick R, Mirza N: Prediction of difficult laryngoscopy: does obesity play a role? Ann Otol Rhinol Laryngol 2007;116:799-804.

21 Weinstein G, O’ Malley BW Jr: daVinci Transoral Surgery Procedure Guide. Intuitive Surgical Training Publication 2011.

-22 Kayhan FT, Kaya KH, Sayin I: Transoral robotic cordectomy for early glottic carcinoma. Ann Otol Rhinol Laryngol 2012;121:497-502.

23 Weinstein GS, O’Malley BW Jr, Snyder W, et al: Transoral robotic surgery: supraglottic partial laryngectomy. Ann Otol Rhinol Laryngol 2007;116:19-23. 